\title{
Low cost production of computer-generated holograms: from design to optical evaluation
}

Ignacio Moreno

i.moreno@umh.es

\section{Antonio Martínez-García}

Lukasz Nieradko

\author{
Jorge Albero \\ Christophe Gorecki
}

\author{
Dept. Ciencia de Materiales, Óptica y Tecnología Electrónica, Universidad Miguel Hernández, Elche, \\ Spain \\ Dept. Ciencia de Materiales, Óptica y Tecnología Electrónica, Universidad Miguel Hernández, Elche, \\ Spain \\ Dépt. MN2S, FEMTO-ST (UMR CNRS 6174), UFR Sciences, 16 Route de Gray, 25030 Besançon cedex, \\ France \\ Wroclaw Research Center EIT+ Stablowicka Str. 147/149 54-066 Wroclaw, Poland \\ Dépt. MN2S, FEMTO-ST (UMR CNRS 6174), UFR Sciences, 16 Route de Gray, 25030 Besançon cedex, \\ France \\ Dépt. MN2S, FEMTO-ST (UMR CNRS 6174), UFR Sciences, 16 Route de Gray, 25030 Besançon cedex, \\ France
}

In this work we review some optical characterization methods useful for the low cost production of two phase level computer generated holograms (CCH). As an example, binary CGH are designed with an iterative Fourier transform algorithm (IFTA) and fabricated on a silicon master micromachining with a single step of selective dry etch of silicon dioxide $\left(\mathrm{SiO}_{2}\right)$ layer. The $\mathrm{CCH}$ characterization is performed in three steps; a first one involves the application of spectroscopic ellipsometry measurements to accurately measure the thickness of the $\mathrm{SiO}_{2}$ layer. These results permit the evaluation of the relative complex reflectance between the two levels of the developed hologram as a function of the wavelength. In a second step, interference microscopy is applied to directly visualize the phase shift in the $\mathrm{SiO}_{2} / \mathrm{Si}_{\text {binary }}$ phase profile. Finally, the performance and diffraction efficiency of the fabricated CGH is compared for various lasers with different wavelengths. These experimental measurements in these two last steps confirm with very good accuracy the results derived from the spectroscopic ellipsometry analysis. In conjunction, the combination of these well established optical techniques provides a precise optical characterization of binary diffractive optical elements produced with simple and low cost technique, useful for mass production. [D0I: 10.2971/jeos.2010.10011]

Keywords: diffraction and scattering, Fourier optics, computer-generated holograms, diffraction efficiency, resolution and other hologram characteristics

\section{INTRODUCTION}

Many different technologies have been used to fabricate computer generated holograms $(\mathrm{CGH})$ and diffractive optical elements (DOE) over a variety of substrates [1]-[3]. Although this is a mature topic, there is still a great interest to develop and apply cost effective fabrication processes, as well as effective tools for the quality evaluation of the fabricated elements $[4,5]$. In general, two types of studies have been conducted in the field: those intended either to improve the fabrication process or to reduce its cost, and those intended to improve the CGH design.

With respect to their fabrication, phase-only CGH have been highly investigated since they are more light efficient. Phase holograms with a continuous phase profile (kinoform) provide higher diffraction efficiencies [6]. However, elements with multiple phase levels usually require a multistep fabrication process, with the consequent disadvantage in terms of time consuming and the strict requirements on multimask alignment and etching accuracy [7]. Therefore, due to its fabrication simplicity and reduced cost, binary phase holograms continue to be very attractive [8]. Many high quality fabrication techniques have been demonstrated, including e- beam lithography $[9,10]$, photolithography [11] or laser ablation $[12,13]$. Requirements for low cost and simple production also led to the application of high resolution laser printers [14, 15]. In all cases, the quality of the fabricated CGH is reflected in the values of the diffraction efficiency [16].

In general, two types of phase CGH designs can be considered: kinoform [6] and detour phase holograms [17]. In kinoform holograms the phase in each pixel is directly generated onto the CGH substrate. For detour phase holograms, the phase of each pixel is encoded through the displacement of the corresponding aperture in the hologram. In this way, detour phase holograms can be encoded on substrates where it is not possible to physically create a phase difference. Kinoform holograms are very efficient in terms of space bandwidth (there is a one-to-one relation between pixels in the designed hologram and pixels in the fabricated hologram), and they can ideally achieve the maximum diffraction efficiency. However, they are rather sensible to possible fabrication errors, like etching depth or surface roughness errors $[18,19]$. On the contrary, detour phase CGH are very exigent in terms of space bandwidth since each pixel of the designed hologram requires a 
matrix of pixels in the fabricated hologram to encode the aperture displacements. They also suffer low diffraction efficiency, both because of the encoding technique, and also because they are usually transferred onto substrates where the optical modulation is produced on the amplitude transmission (although they can be also converted onto phase holograms, see [20]). On the contrary, they present better tolerance to fabrication errors, since they depend on the precise location of the lateral in plane displacements rather than the precise etching depths. The efficiency of detour phase holograms can also be improved by combining them with carrier gratings [21].

In this work, we review the application of some well established techniques useful to produce and accurately characterize binary phase reflective Fourier transform CGH fabricated over silicon substrate. In spite of its poor optical properties, silicon offers attractive possibilities for combination with high quality materiales [22, 23]. Here, the inclusion of micromachining steps offers an accessible mass production method that minimizes fabrication complexity, component turnaround time, and cost. All these advantages are obtained by transferring the designed binary phase holograms onto a silicon wafer with a thermal oxide $\left(\mathrm{SiO}_{2}\right)$ layer. The two required phase levels are obtained by selectively etching the $\mathrm{SiO}_{2}$ layer with the hologram pattern, resulting in a reflective binary phase hologram. The phase difference between the two levels of the reflective $\mathrm{CGH}$ is the key parameter to achieve good diffraction efficiency, and inspection tools useful to characterize this phase shift can be very useful in the production process.

Here, the physical phase difference is produced by the optical path difference between the light reflected on the $\mathrm{SiO}_{2} / \mathrm{Si}$ and the light directly reflected on the $\mathrm{Si}$ surface $\left(\mathrm{SiO}_{2}\right.$ layer removed). But additionally, thin film interference additionally contributes to the phase difference, and also produces a significant difference in the amplitude reflection coefficients, thus affecting the diffraction efficiency. Therefore, an accurate characterization of this thin film interference can provide valuable information. Spectroscopic ellipsometry is an optical technique useful for such purpose [24]. In this work, we apply variable angle spectroscopic ellipsometry (VASE) to precisely determine the thickness of the deposited $\mathrm{SiO}_{2}$ layer, and the complex reflection coefficients at the two levels in the fabricated CGH. With this information it is possible to accurately estimate the diffraction efficiency in the whole spectral range covered by the ellipsometer (in our case from $370 \mathrm{~nm}$ to $1000 \mathrm{~nm}$ ). This analysis is further completed with the application of interference microscopy [25] to measure the phase difference between the two levels generated on the hologram. Images captured with a microscope incorporating an interferometric objective confirm the results derived from the ellipsometric measurements and visualize the wavelength ranges where the fabricated CGH show best diffraction efficiency. We finally present measurements of the diffraction efficiency for three available wavelengths and provide experimental results on the CGH reconstruction that verify the results derived from the ellipsometric analysis. In conjunction, we present a full optical characterization of the fabricated CGH, showing useful inspection tools to determine the quality of the fabrication procedure.
The paper is organized as follows. In Section 2, we describe the steps followed to design the holograms, and we describe the fabrication process. In Section 3, we present the results obtained from the application of the spectroscopic ellipsometry analysis of the $\mathrm{SiO}_{2} / \mathrm{Si}$ layer, as well as the visual inspection of the fabricated CGH by means of interference microscopy. In Section 4, we present the calculation of the diffraction efficiency of the fabricated holograms, its wavelength dependence derived from the previous analysis, and the experimental reconstructions for three different wavelengths. Finally, Section 5 presents the conclusions of the work.

\section{FABRICATION PROCEDURE AND CGH DESIGN}

The design of $\mathrm{CGH}$ is a subject that has been studied for more than forty years and a variety of techniques have been developed [3, 4]. Here we have considered two fabrication limitations. We want to generate the CGH in a single step and therefore we consider binary phase-only holograms. Secondly, in order to avoid too large computer memory and time requirements, we limited the hologram masks to square images with 1024 pixels $\times 1024$ pixels. We designed a CGH to reconstruct the text "FEMTO-MOEMS" (see Figure 1(a)) which is slightly displaced from the centre in the vertical direction, to avoid overlapping of the reconstruction with the DC peak. We applied an iterative inverse Fourier transform algorithm (IFTA) $[26,27]$ to calculate the phase mask to be transferred onto the CGH. Following [28], the modulus of the CGH complex amplitude distribution is set to unity in the Fourier domain, and the modulus of the complex amplitude distribution at the reconstruction plane is set to the original one on the image domain. A window is defined around the reconstruction area in this last case, allowing amplitude and phase freedom for the light outside this window. In order to obtain a rapid convergence, we added a random phase to the original image in the first iteration. We applied 30 iterations of this procedure, only the last fifteen applying the reconstruction window constrain, so that a good balance between signal to noise

\section{FEMTO (a) MOEMS}

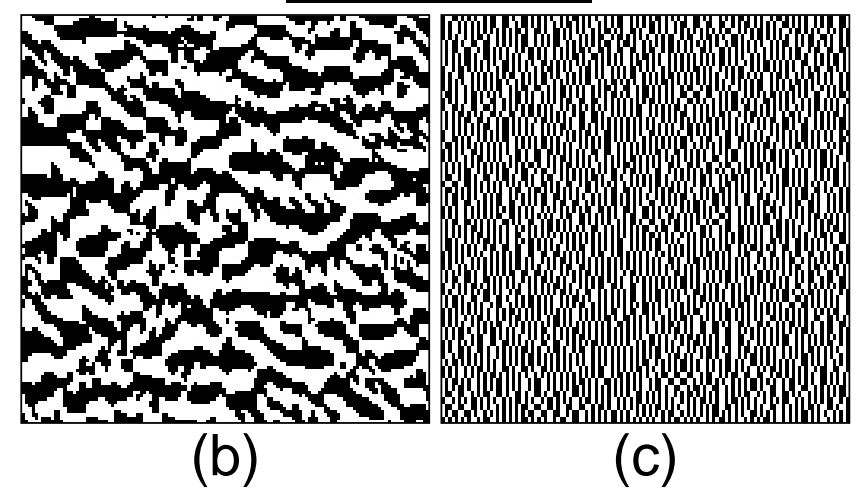

FIG. 1 (a) Object to encode onto the holograms. Detailed areas of the binary phase masks designed to act as: (b) Direct phase (kinoform) $\mathrm{CGH}$, and (c) Detour phase CGH. 


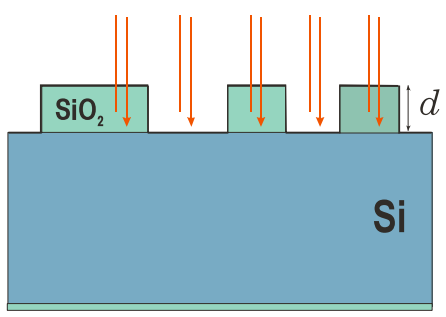

(a)

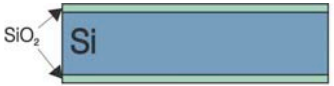

1st. Hard mask over Si: Thermal $\mathrm{SiO}_{2}$

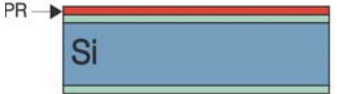

2nd. Spin coating of the substrate with positive photoresist (PR)

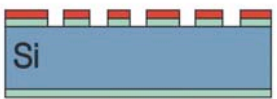

3rd. Photolithography and aperture of the pattern on the mask with $\mathrm{BHF}$

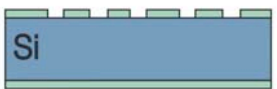

4th. Removal of photoresist and cleaning

\section{(b)}

FIG. 2 (a) Scheme for the reflective hologram efficiency design. $d$ is the physical depth between the two regions in the hologram. (b) Hologram fabrication steps.

ratio and fidelity of the reconstruction is obtained. The final phase distribution in the Fourier domain is then binarized, assigning phase values 0 and $\pi$ to phase ranges $[0, \pi)$ and $[\pi, 2 \pi)$, respectively. For comparison, we also implemented the detour phase technique. Although many different encoding techniques have proposed [29], here we use for simplicity the original Lohmann's technique [17]. It allows encoding multiple phase levels, but since we want to compare results with those from the binary kinoform, we again considered the binary hologram and used only two displacement levels. Therefore, we restrict the hologram size to the central 512 pixels $\times 512$ pixels, and each pixel of the original hologram is converted onto a cell of 2 pixels $\times 2$ pixels. The aperture is located on the left for phase 0 and on the right for phase $\pi$. Figures $1(\mathrm{~b})$ and 1 (c) show a detail of these two designed CGH, where black and white denote phases 0 and $\pi$ respectively.

The procedure for $\mathrm{CGH}$ fabrication is based on selectively etching a $\mathrm{SiO}_{2}$ layer grown on top of a silicon wafer, which acts as the reflective surface. This non typical technique for the production of diffractive elements was already used in [30]. The silicon micromachining technology employed here, in particular the silicon etching, is well suitable to fabricate the designed precision-defined micro-optical components, offering relative easy procedures and presenting a potential use for monolithic integration at low cost. Therefore, this simple hologram fabrication process could be suitable for mass production at low cost, and it can be easily combined with conventional techniques of replication from silicon master [31].

Figure 2(a) shows a scheme of the processed reflective mask. The holograms are fabricated using well known micromachining processes. Monocrystalline silicon is used as substrate and a $\mathrm{SiO}_{2}$ layer is grown by thermal oxidation (see Figure 2(b), $1^{\text {st }}$ step). Positive photoresist is spin coated over the wafer (see Figure 2(b), $2^{\text {nd }}$ step) and the pattern is transferred by direct laser writing with a Heidelberg DLW200 instrument. After exposure to the laser writing, the photoresist is developed and hologram pattern is then etched on the $\mathrm{SiO}_{2}$ layer using a solution of buffered hydrofluoridic acid (BHF) (see Figure 2(b), $3^{\text {rd }}$ step). Once the $\mathrm{SiO}_{2}$ layer is patterned, the photoresist is removed using acetone and the substrate is cleaned with piranha (mixture of $\mathrm{H}_{2} \mathrm{O}_{2}+\mathrm{H}_{2} \mathrm{SO}_{4}$, see Figure 2(b), $4^{\text {th }}$ step) and rinsed in deionized water. The $\mathrm{CGH}$ mask to be transferred to the wafer was designed using commercially available CAD software. Each pixel was treated as an independent square which, depending on the designed phase value, corresponds to an absence (hollow volume) or presence of $\mathrm{SiO}_{2}$.

In general, the energy loss at the $\mathrm{SiO}_{2} / \mathrm{Si}$ profile could be reduced by covering it with a high reflection metal film [30]. Our purpose here, however, is not to develop the final CGH product, but to obtain a good quality $\mathrm{CGH}$ master, that can be further replicated for instance onto polymer [31]. Therefore metallization of the samples is not necessary. This provides non optimized diffraction efficiency on this fabricated master $\mathrm{CGH}$. The focus of this paper is, instead, on the demonstration of the usefulness of the two above mentioned optical inspection methods (spectroscopic ellipsometry and interference microscopy), as very accurate tools to determine the complex reflectance of the fabricated $\mathrm{SiO}_{2} / \mathrm{Si} \mathrm{CGH}$, in order to calculate the diffraction efficiency.

\section{SPECTROSCOPIC ELLIPSOMETRY CHARACTERIZATION AND MICROSCOPY INSPECTION}

Spectroscopic ellipsometry is a very well established technique that allows the determination of the optical properties of thin film layers, (optical constants of the material and thickness of the layer) by measuring the changes in the polarization ellipse on the broadband light reflected from the sample at different incidence angle [24]. We use here a J. A. Woollam Co. M2000V-RCE variable angle spectroscopic ellipsometer (VASE) [32], which can measure at different angles of incidence for 390 different wavelengths in the range from $370 \mathrm{~nm}$ to $1000 \mathrm{~nm}$. This technique has been proven to be very accurate for the characterization of $\mathrm{SiO}_{2} / \mathrm{Si}$ samples [33]. Here, we captured experimental ellipsometric data of the processed $\mathrm{SiO}_{2} / \mathrm{Si}$ samples by illuminating an area of the wafer that has not been etched with the CGH elements.

The standard ellipsometric parameters, $\Psi$ and $\Delta$ are related to the complex ratio $\rho$ of the reflection coefficients for light polarized parallel $(p)$ and perpendicular $(s)$ to the plane of incidence, defined as [24]

$$
\rho=\frac{r_{p}}{r_{s}}=\tan (\Psi) \exp (i \Delta) .
$$

Figures 3(a) and 3(b) show the experimentally captured data for three angles of incidence $\left(60^{\circ}, 65^{\circ}\right.$ and $\left.70^{\circ}\right)$. They show, respectively, the ellipsometric angles $\Psi(\lambda)$ and $\Delta(\lambda)$, as a function of the wavelength. These experimental data are then fitted to numerical equivalent curves calculated from the reflection coefficients of the $\mathrm{SiO}_{2} / \mathrm{Si}$ sample. Figure 4 shows the 

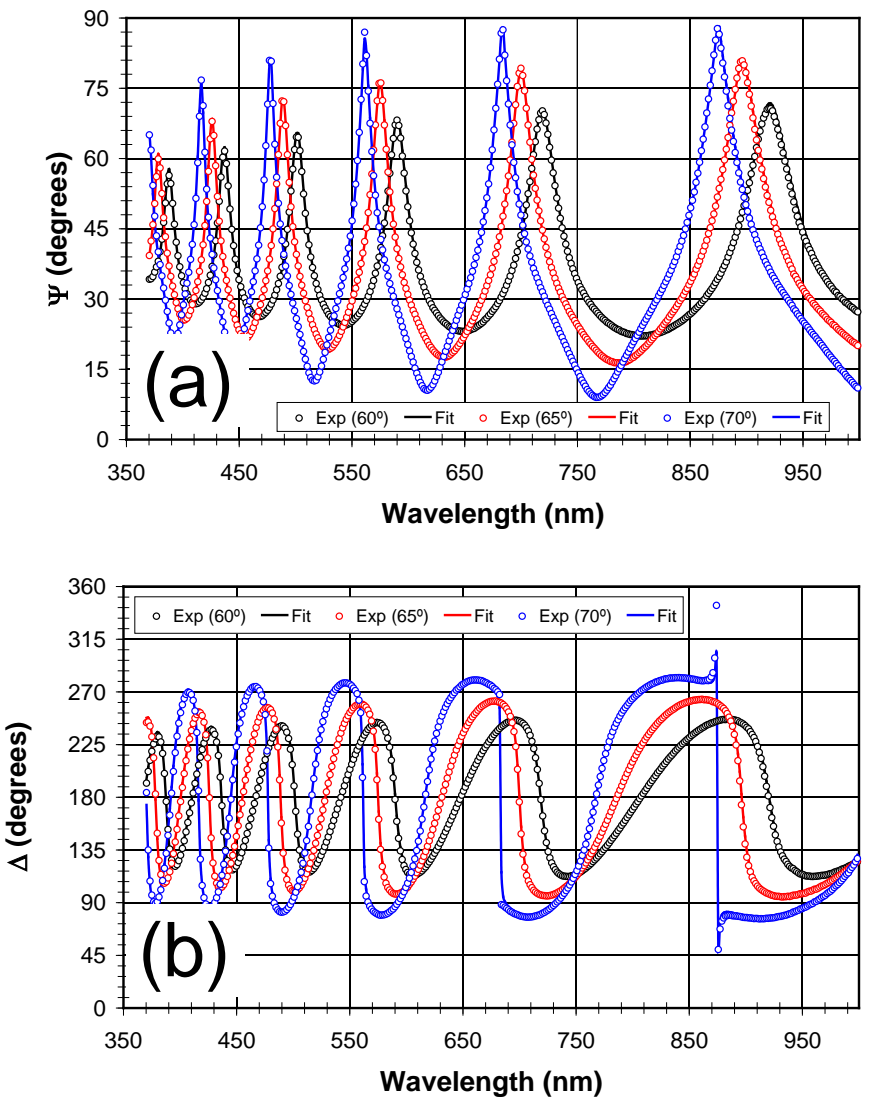

FIG. 3 Measured and fitted ellipsometric angles versus wavelength for the $\mathrm{SiO}_{2}$ layer over Si substrate for three incidence angles $\left(60^{\circ}, 65^{\circ}\right.$, and $\left.70^{\circ}\right)$ : (a) $\Psi(\lambda)$, (b) $\Delta(\lambda)$.

optical constants for $\mathrm{Si}$ and $\mathrm{SiO}_{2}$ selected from the materials database in the ellipsometer software, which have been used in the calculations $\left(\mathrm{SiO}_{2}\right.$ can be considered transparent in the selected range, and $k=0$ has been selected in this case). The simultaneous fit of all the six curves presented in Figure 3 permit a precise determination of the thickness of the deposited $\mathrm{SiO}_{2}$ layer. The best fit is obtained for a $\mathrm{SiO}_{2}$ layer thickness of $1382.6 \mathrm{~nm}$.

Then, once the thickness of the $\mathrm{SiO}_{2}$ layer and the optical constants are know, it is possible to predict the relative complex reflectance between the light reflected on top of the $\mathrm{SiO}_{2}$ layer compared to the light reflected directly on $\mathrm{Si}$ after selective removal of the $\mathrm{SiO}_{2}$ layer. Figure 5 shows the corresponding results. A first intuitive approximation is given by the optical path difference caused by the different refractive indices of $\mathrm{SiO}_{2}$ and air. With this initial approximation, the phase shift $(\Delta \phi)$ between the light reflected on the two levels in the fabricated CGH will be given by

$$
\Delta \phi_{\mathrm{OPD}}=\frac{4 \pi d}{\lambda}\left(n_{\mathrm{SiO}_{2}}-1\right)
$$

where $\lambda$ stands for the wavelength of the incoming light, $n_{\mathrm{SiO}_{2}}$ is the $\mathrm{SiO}_{2}$ refractive index, and $d$ is the thickness of the $\mathrm{SiO}_{2}$ layer. The curve $\Delta \phi_{\mathrm{OPD}}(\lambda)$, derived from Eq. (2) for the values $n_{\mathrm{SiO}_{2}}(\lambda)$ shown in Figure 4 and the thickness $d=1382.6 \mathrm{~nm}$, is presented in Figure 5 as the optical path difference(OPD) phase curve. In this figure, we indicated the three wavelengths $488 \mathrm{~nm}, 543 \mathrm{~nm}$ and $633 \mathrm{~nm}$, which correspond to three laser lines that we employ for the reconstruction of the fabricated CGH. We can see that the expected phase shift for $633 \mathrm{~nm}$

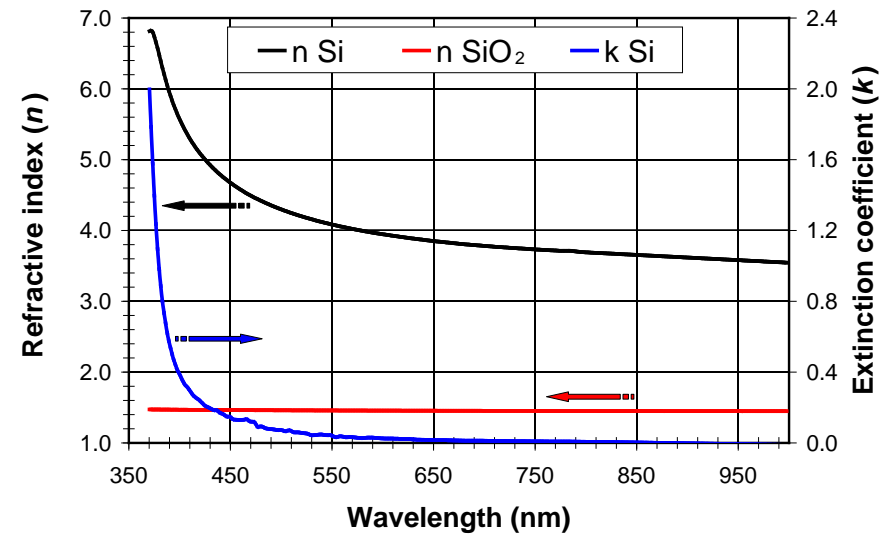

FIG. 4 Optical constants (refractive index and extinction coefficient) for $\mathrm{Si}$ and $\mathrm{SiO}_{2}$.
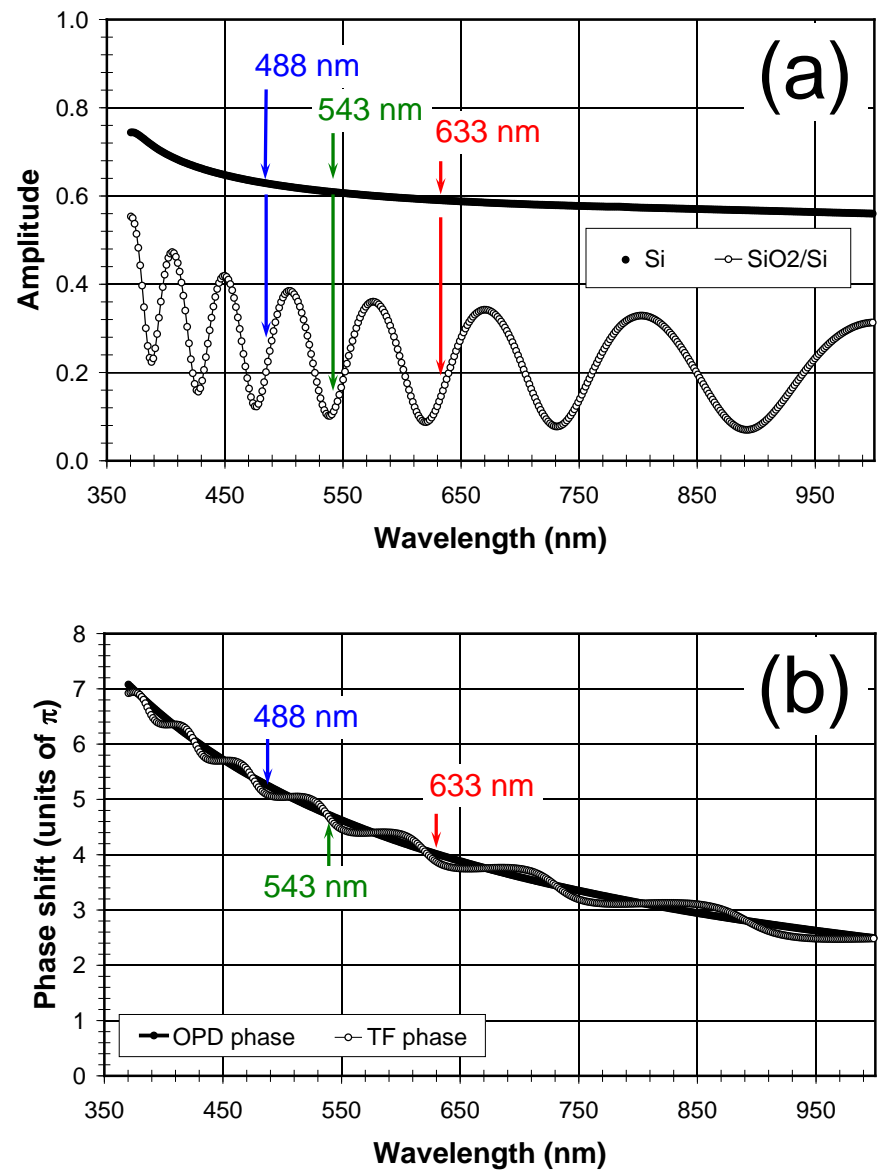

FIC. 5 Complex amplitude reflection coefficient versus wavelength at the two surfaces ( $\mathrm{Si}$ and $\mathrm{SiO}_{2} / \mathrm{Si}$ ) in the fabricated $\mathrm{CCH}$ calculated from ellipsometric data. (a) Modulus of the reflection coefficient, (b) Relative phase shift between the light reflected at the two surfaces, calculated as the optical path difference (OPD), or through the thin film (TF) analysis.

is very close to be $4 \pi$ radians, while it is relatively close to $5 \pi$ radians for $543 \mathrm{~nm}$ and $488 \mathrm{~nm}$. The maximum diffraction efficiency will be obtained when $\Delta \phi$ is an odd multiple of $\pi$ radians. Therefore, a rather reduced efficiency is expected for $633 \mathrm{~nm}$, while better efficiency is expected for $543 \mathrm{~nm}$ and $488 \mathrm{~nm}$.

This approximated analysis, however, must be refined to take into account the reflection coefficients on the two surfaces on the CGH, which are complex valued and therefore add addi- 
tional phases. This is especially relevant in the reflection from the $\mathrm{SiO}_{2} / \mathrm{Si}$ layer, due to the thin film interference. The reflection coefficient at the air-SiO $2 / \mathrm{Si}$ layer $\left(r_{012}\right)$ is given by the thin film interference [24],

$$
r_{012}=\frac{r_{01}+r_{12} \exp (-i 2 \beta)}{1+r_{01} r_{12} \exp (-i 2 \beta)}
$$

where $r_{01}$ and $r_{12}$ are the Fresnel reflection coefficients at the air- $\mathrm{SiO}_{2}$ interface and at the $\mathrm{SiO}_{2}-\mathrm{Si}$ interface respectively,

$$
r_{01}=\frac{1-N_{\mathrm{SiO}_{2}}}{1+N_{\mathrm{SiO}_{2}}}, \quad r_{12}=\frac{N_{\mathrm{SiO}_{2}}-N_{\mathrm{Si}}}{N_{\mathrm{SiO}_{2}}+N_{\mathrm{Si}}},
$$

and

$$
\beta=\frac{2 \pi}{\lambda} N_{\mathrm{SiO}_{2}} d
$$

The reflection coefficient at the air-Si interface $\left(r_{02}\right)$ is

$$
r_{02}=\frac{1-N_{\mathrm{Si}}}{1+N_{\mathrm{Si}}}
$$

In these equations, $N$ stands for the complex refractive index, thus being $N_{\mathrm{SiO}_{2}}=n_{\mathrm{SiO}_{2}}$ and $N_{\mathrm{Si}}=n_{\mathrm{Si}}-i k_{\mathrm{Si}}$. If these reflection coefficients are written in polar form, $r_{012}=$ $\left|r_{012}\right| \exp \left(i \phi_{012}\right)$ and $r_{02}=\left|r_{02}\right| \exp \left(i \phi_{02}\right)$, the phase difference of the light reflected at the two surfaces in the fabricated $\mathrm{CGH}$ can be calculated as

$$
\Delta \phi_{T F}=\phi_{012}-\left(\phi_{02}+\frac{4 \pi}{\lambda} d\right)
$$

where $4 \pi d / \lambda$ stands for the phase gained by the wave propagated in air a distance twice the thickness of the $\mathrm{SiO}_{2}$ layer.

Figure 5 shows the results obtained with these equations when the optical constants presented in Figure 4 are employed. Figure 5(a) shows the dependence with wavelength of the modulus of the two reflection coefficients, $\left|r_{02}\right|$ and $\left|r_{012}\right|$, showing the last one the characteristic thin film oscillatory behavior. Figure $5(\mathrm{~b})$ shows the wavelength dependence of the thin film (TF) phase difference calculated from Eq. (7), which shows also an oscillation around the OPD phase calculated from Eq. (2). Table 1 gives the values for the modulus and phase difference calculated for the three selected wavelengths.

\begin{tabular}{|c|c|c|c|}
\hline Wavelength (nm) & $\left|r_{02}\right|$ & $\left|r_{012}\right|$ & $\Delta \phi_{\mathrm{TF}}$ \\
\hline 488 & 0.63 & 0.25 & $5.08 \pi$ \\
\hline 543 & 0.61 & 0.12 & $4.56 \pi$ \\
\hline 633 & 0.59 & 0.15 & $3.84 \pi$ \\
\hline
\end{tabular}

TABLE 1 Modulus of the reflection coefficients and relative phase at the two regions of the CGH for the three selected wavelengths.

Next, in order to confirm these results, we inspected the fabricated CGH with a Nikon LV100-Pol microscope. Images were captured with a Nikon DCFi1 CCD camera. Figure 6 shows some of these images captured using a $20 \times$ microscope objective, corresponding to a binary grating, and the kinoform and detour phase CGHs shown in Figure 1. All three elements were fabricated onto the same wafer with the same conditions, and using a nominal pixel size of $3 \mu \mathrm{m}$. The binary grating in

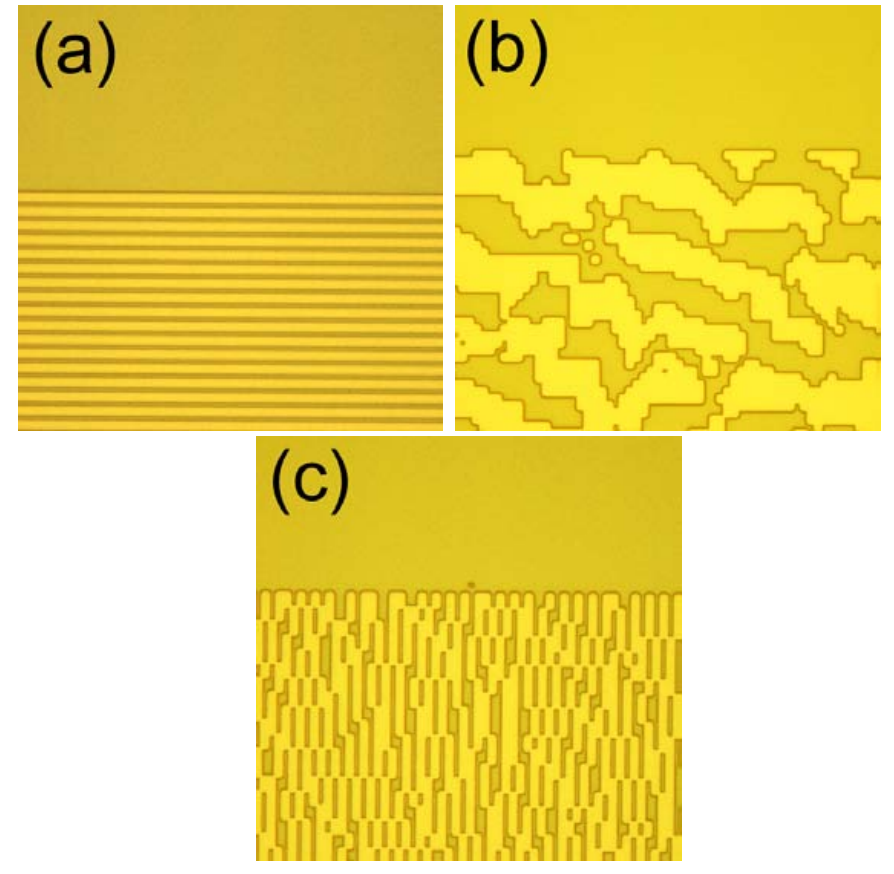

FIC. 6 Microscope images of the fabricated elements: (a) Binary diffraction grating, (b) kinoform CGH, and (c) Detour phase CGH.

Figure 6(a) is designed with maximal spatial resolution (one pixel white and one pixel black). While the grating is very well reproduced, it shows a non perfect $50 \%$ fill factor (ratio between the size of one grating level to the grating's period). Instead a value of approximately $26 \%$ is measured. Figures $6(\mathrm{~b})$ and $6(\mathrm{c})$ show microscope images captured in the same conditions for the two CGH designs. In all these images we selected an area where a uniform part is also visible, since it is very useful for characterization of the phase depth when we use the interference objective, as we show next.

Interference microscopy is a useful technique to measure depth changes as shifts in the interference fringes captured at the microscope [34, 35]. Figure 7 shows the interference patterns corresponding to the binary grating shown in Figure $6($ a) captured with a $20 \times$ differential interference objective from Nikon. Figure 7(a) shows the image captured in the microscope when the broadband illumination is selected. The grating is focused and the microscope platform tilt is adjusted to obtained interference fringes perpendicular to the border between the uniform region and the grating region (which is also oriented with the slits perpendicular to the interference fringes). Figure 7(a) shows a clear shift in the polychromatic interferogram on the top of the image (which corresponds to the uniform area where $\mathrm{SiO}_{2}$ is not removed) in comparison with the interferogram on the bottom of the image, which corresponds to the grating area. A shift of more than two fringes is clearly visible for the whole broadband spectrum. Figures 7(b), 7(c) and 7(d) show the corresponding image when an interference filter is introduced in the microscope, with transmissions at $488 \mathrm{~nm}, 540 \mathrm{~nm}$ and $633 \mathrm{~nm}$, respectively. In each of these images we added an arrow indicating the fringe shift expected from the phases presented in Table 1 calculated from the ellipsometric analysis. In all cases, we see an excellent agreement. The blue image shows a shift of the fringes is very close to 2.5 periods, while it is clearly 

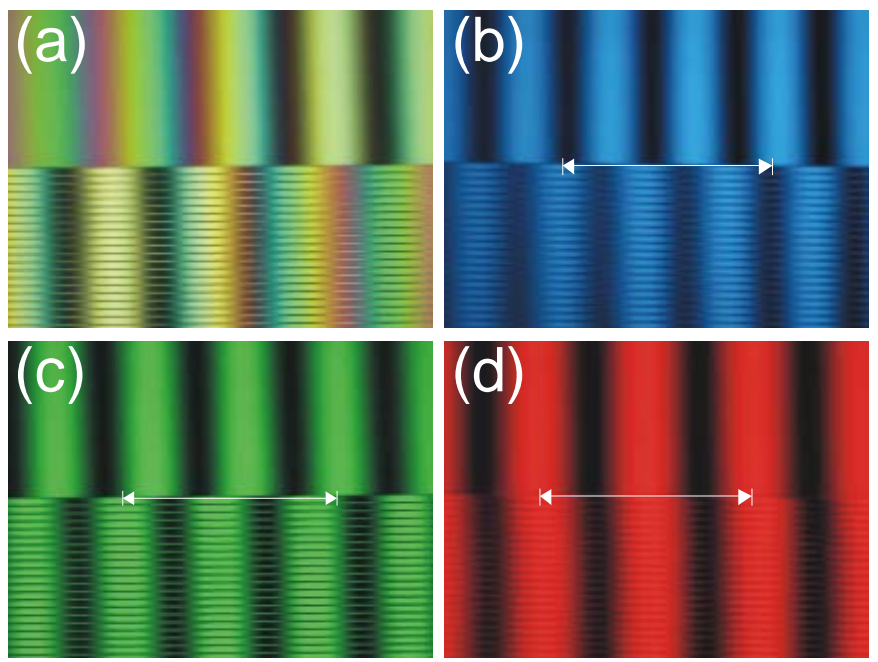

FIC. 7 Microscope images of the binary diffraction grating captured with the interferometric objective. (a) Broadband illumination, (b) $488 \mathrm{~nm}$, (c) $540 \mathrm{~nm}$, (d) $633 \mathrm{~nm}$

less than 2.5 periods for the green image, and very close to two periods for the red image.

This combined ellipsometric and microscopic analysis does not provide an accurate measurement on the borders between the two levels in the hologram. For that purpose other more expensive techniques such as optical metrology [36], scanning electron microscopy (SEM) [37,38] or atomic force microscopy (AFM) [39] have been typically used. However, as we show next, the approach followed here where the reflection coefficients are directly measured, provides relevant information useful to predict, with rather good accuracy, the diffraction efficiency of the produced elements.

\section{DIFFRACTION EFFICIENCY AND EXPERIMENTAL RECONSTRUCTION}

The good agreement of the phases predicted from the ellipsometric analysis and measured in the microscope suggest that the reflection coefficients presented in Figure 5 can be used to calculate the diffraction efficiency in the whole spectral range characterized with the ellipsometer. For that purpose, we adopt a simple model based on scalar diffraction theory, that incorporates two regions in the CGH, with reflection magnitudes $r_{1}$ and $r_{2}$ (in this case $r_{1} \equiv\left|r_{02}\right|$ and $r_{2} \equiv\left|r_{012}\right|$ ) and relative phase shift $\phi$ between them (in this case $\phi=\Delta \phi_{\mathrm{TF}}$ ), as well as a fill factor described with a parameter $a$ equal to the ratio between the width of the first level and the period of the grating [40]. The grating reflectivity function can be described mathematically as the function $g(x)$ defined as:

$$
\begin{aligned}
& g(x)= \\
& \left\{r_{1} \operatorname{rect}\left(\frac{x}{p}\right)+\left(r_{2} e^{i \phi}-r_{1}\right) \operatorname{rect}\left(\frac{x}{a p}\right)\right\} \otimes \sum_{m} \delta(x-m p)
\end{aligned}
$$

where $x$ is the spatial coordinate, $p$ denotes the period of the grating, $a$ is the fill factor (for the ideal $50 \%$ fill factor grating $a=1 / 2$ ), the symbol $\otimes$ indicates the convolution operation, $m$ are integer numbers, and the function $\operatorname{rect}(\cdot)$ is defined as [41]

$$
\operatorname{rect}(x)=\left\{\begin{array}{cc}
1 & \text { if }|x|<1 / 2 \\
1 / 2 & \text { if }|x|=1 / 2 \\
0 & \text { if }|x|>1 / 2
\end{array} .\right.
$$

The diffraction pattern generated by the grating is evaluated by Fourier transforming the grating function $g(x)$ in Eq. (8), leading to

$$
\begin{aligned}
G(u) & \equiv \operatorname{FT}\{g(x)\} \\
& =\left\{r_{1} \operatorname{sinc}(p u)+a\left(r_{2} e^{i \phi}-r_{1}\right) \operatorname{sinc}(a p u)\right\} \sum_{m} \delta\left(u-\frac{m}{p}\right) \\
& =\sum_{m} G_{m} \cdot \delta\left(u-\frac{m}{p}\right)
\end{aligned}
$$

where FT $\{$.$\} denotes the Fourier transform operation, u$ is the spatial frequency, and $\operatorname{sinc}(u)=\sin (\pi u) /(\pi u)$. The amplitude $G_{m}$ of each $m$ diffraction order is given by

$$
G_{m}=r_{1} \operatorname{sinc}(m)+a\left(r_{2} e^{i \phi}-r_{1}\right) \operatorname{sinc}(a m),
$$

and the corresponding intensities are given by $i_{m}=\left|G_{m}\right|^{2}$. The important terms related to the CGH efficiency are the $m= \pm 1$ orders, that correspond to the direct and inverted hologram reconstructions, and the $m=0$ order, that corresponds to the DC peak appearing on axis [42]. The intensities $i_{0}$ and $i_{ \pm 1}$, given in terms of the relative phase shift $\Delta \phi$ and the fill factor $a$, are

$$
\begin{gathered}
i_{0}=\left|G_{0}\right|^{2}=r_{1}^{2}(1-a)^{2}+a^{2} r_{2}^{2}+2 r_{1} r_{2} a(1-a) \cos (\phi), \\
i_{ \pm 1}=\left|G_{ \pm 1}\right|^{2}=a^{2} \operatorname{sinc}^{2}(a)\left[r_{1}^{2}+r_{2}^{2}-2 r_{1} r_{2} \cos (\phi)\right] .
\end{gathered}
$$

These equations reveal that, for a given fixed value of the magnitude of the reflected coefficients $\left(r_{1}\right.$ and $\left.r_{2}\right)$, and for a fixed value of the fill factor $a$, the maximum diffraction efficiency (maximum value of $i_{ \pm 1}$ ) is always obtained when $\phi=\pi$, reaching then values $i_{ \pm 1}^{\max }=a^{2} \operatorname{sinc}^{2}(a)\left(r_{1}+r_{2}\right)^{2}$ and $i_{0}^{\min }=\left(r_{1}(1-a)-r_{2} a\right)^{2}$. In the ideal case where $r_{1}=r_{2}=1$ and the fill factor is $a=1 / 2$, they become $i_{ \pm 1}^{\max }=0.405$ and $i_{0}^{\min }=0$.

Figure 8 shows the expected results for $i_{0}$ and $i_{ \pm 1}$ as a function of the wavelength, calculated from Eqs. (12) with the complex amplitude reflectance values presented in Figure 5, and the observed fill factor $a=0.26$. We have tested the diffraction gratings and the holograms response with three lasers with different wavelengths: the blue line $(488 \mathrm{~nm})$ from a multiline argon ion laser, and two He-Ne lasers with wavelengths $543 \mathrm{~nm}$ and $633 \mathrm{~nm}$, respectively. We measured the diffraction efficiency of the gratings by measuring the intensities of the zero and first diffraction orders normalized to the intensity of the incident beam. The results are marked as the square and triangular points on the figure, showing an excellent agreement with the predictions. Figure 8 shows that the diffraction efficiency is relatively low at the whole spectral range, due to the losses on reflection. However, as we mentioned in the introduction, it is not the goal of this work to develop a final high efficient $\mathrm{CGH}$, but to show the procedure to accurately determine the complex reflection, and its implication in the final efficiency.

Finally, we show the results on the hologram reconstruction. Figure 9 shows the numerical simulation of the reconstruction 


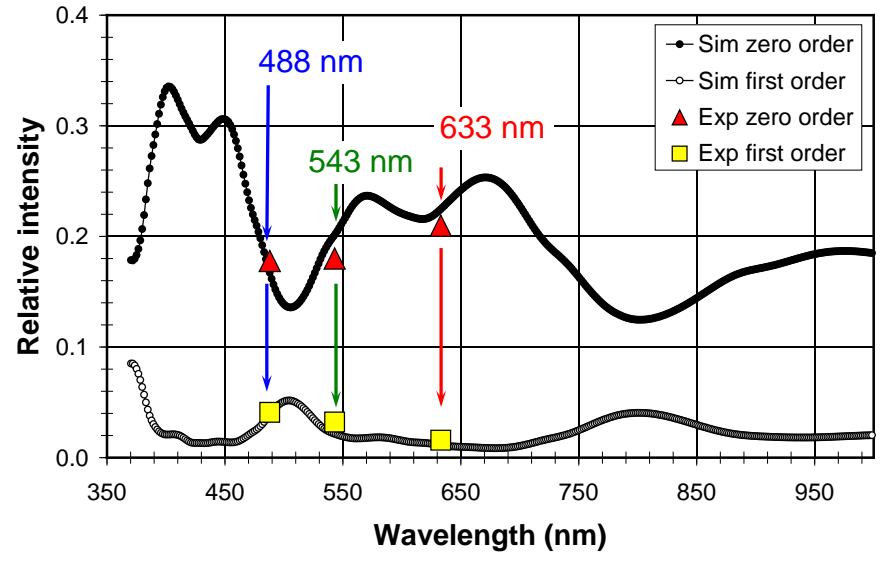

FIC. 8 Relative intensities of the zero and first diffraction orders as a function of the wavelength. The curves indicate the calculated results derived from the data in Figure 5 . The spots indicate the experimental measurements obtained from the diffraction gratings at the three selected wavelengths.

obtained for the kinoform and for the detour phase CGH in Figure 1. In these simulations we consider perfect CGHs with phase levels 0 and $\pi$. Figure 9(a) corresponds to the kinoform hologram. It shows that the words FEMTO-MOEMS are very clearly reconstructed, in the middle of the defined window, outside of which some noise appears. Because of the hologram binarization, an inverted replica of the text is also present, but displaced in opposite sense, thus avoiding overlapping with the direct reconstructions. Figure 9(b) shows the numerical reconstruction from the detour phase CGH. In this case, because of the encoding technique, the reconstruction appears off axis, centered at the \pm 1 diffraction orders. In each order the direct and inverted reconstruction is present since the encoded hologram is binary. Finally, Figure 9(c) shows a detail of the $\mathrm{CGH}$ reconstruction where we indicated three different windows used to evaluate the quality of the produced CGH [14]: window $R$ corresponds to desired signal, i.e., the letters in the text FEMTO-MOEMS, while windows $N_{\text {in }}$ and $N_{\text {out }}$ are used to measure the noise levels inside and outside the reconstruction window. Table 2 gives the mean intensity value and its standard derivation in each of these three windows. All values are normalized to the mean intensity $(i)$ at the reconstruction window $R$. The standard deviation $(\sigma)$ in the window $R$ is a measurement of the uniformity in the reconstruction. These data show that in general the kinoform CGH provides a better performance than the detour phase CGH.

Figure 10 shows the experimental results obtained with the produced CGH. Figures 10(a)-10(c) show the central area obtained with the kinoform CGH for the three selected wavelengths (488 nm, $543 \mathrm{~nm}$ and $633 \mathrm{~nm}$ ). In each case, an excellent reconstruction of the FEMTO-MOEMS text is obtained in agreement with the simulation in Figure 9(a). However, a zero (DC) peak is also present on axis, located in between the two text reconstructions, due to the limited diffraction efficiency. This peak is especially strong for $633 \mathrm{~nm}$ (see Figure 10(c)), in agreement with the predictions in Figure 8. For the green and blue lasers, the efficiency is better and a very good hologram reconstruction is clearly seen. Somehow, the fabricated hologram can be considered as a wavelength-selective hologram in the sense introduced in [43]. Figure 10(d) shows an
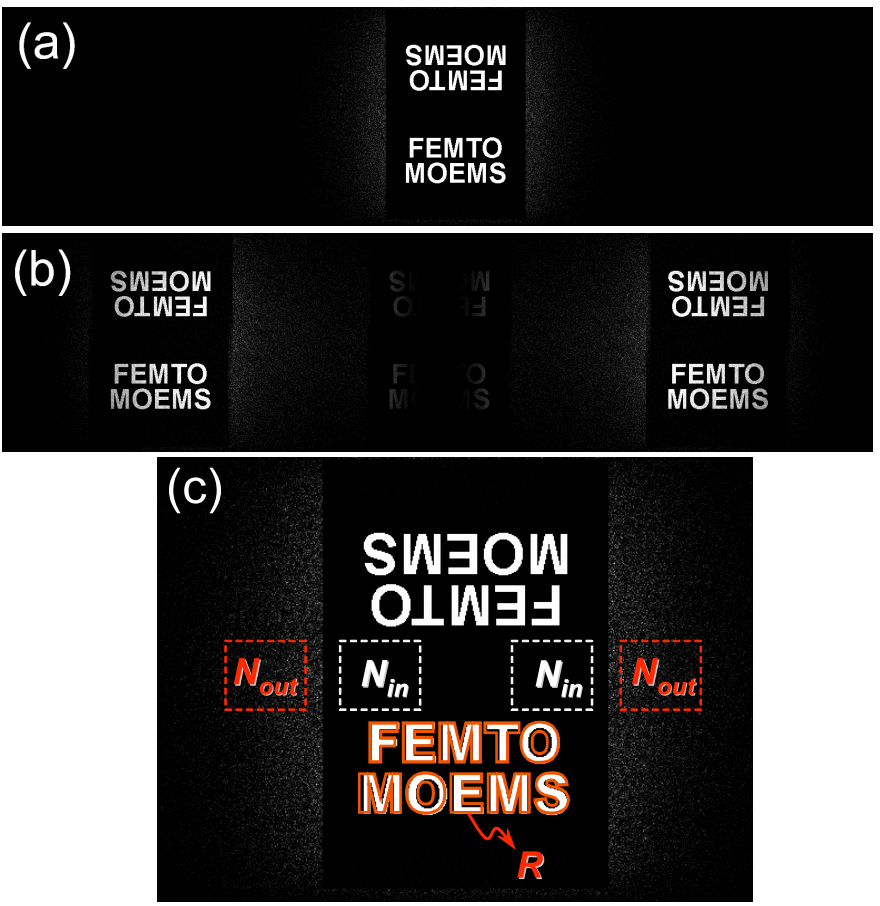

FIG. 9 Numerical simulation of the ideal $\pi$-phase $\mathrm{CCH}$ reconstruction: (a) kinoform $\mathrm{CCH}$, (b) detour phase $\mathrm{CCH}$. (c) Detail where the evaluation windows are indicated. $R$ denotes the item reconstruction window, whereas $N_{\text {in }}$ and $N_{\text {out }}$ denote the noise windows.

\begin{tabular}{|c|c|c|c|c|c|}
\hline CGH & $\begin{array}{c}\text { Evaluation } \\
\text { window }\end{array}$ & $i_{\text {sim }}$ & $\sigma_{\text {sim }}$ & $i_{\exp }$ & $\sigma_{\exp }$ \\
\hline \multirow{3}{*}{ Kinoform } & $R$ & 1 & 0.00472 & 1 & 0.0028 \\
\cline { 2 - 6 } & $N_{\text {in }}$ & 0.002 & 0.00001 & 0.2 & 0.0058 \\
\cline { 2 - 6 } & $N_{\text {out }}$ & 0.062 & 0.00334 & 0.6 & 0.0163 \\
\hline \multirow{2}{*}{$\begin{array}{c}\text { Detour } \\
\text { phase }\end{array}$} & $R$ & 1 & 0.01570 & 1 & 0.0061 \\
\cline { 2 - 6 } & $N_{\text {in }}$ & 0.015 & 0.00013 & 0.2 & 0.0033 \\
\cline { 2 - 6 } & $N_{\text {out }}$ & 0.090 & 0.00506 & 0.4 & 0.0073 \\
\hline
\end{tabular}

TABLE 2 Comparison of the simulated and experimental CGH reconstructions through the mean value and standard deviation at evaluation windows $R, N_{\text {in }}$ and $N_{\text {out }}$. Experimental values correspond to the reconstruction with $\lambda=488 \mathrm{~nm}$.

extended view of the reconstruction area for $488 \mathrm{~nm}$, showing how the reconstruction appears close on axis, while Figure 10(e) shows the corresponding result obtained with the detour phase holograms, again for $488 \mathrm{~nm}$. In both cases the agreement with the numerical simulations in Figures 9(a) and 9(b) is excellent, showing the fidelity of the text reconstructed from the CGH. Table 2 provides the corresponding values for these experimental images of the mean value and the standard deviation in the three evaluation windows defined in Figure 9. As expected, the experimental images show worse values of noise due to possible imperfections in the production, but also because of the speckle originated from the coherent illumination.

\section{CONCLUSIONS}

In summary, we reviewed the different stages of the CGH production and we presented results of the complete process for its development and accurate characterization, including 1) an 

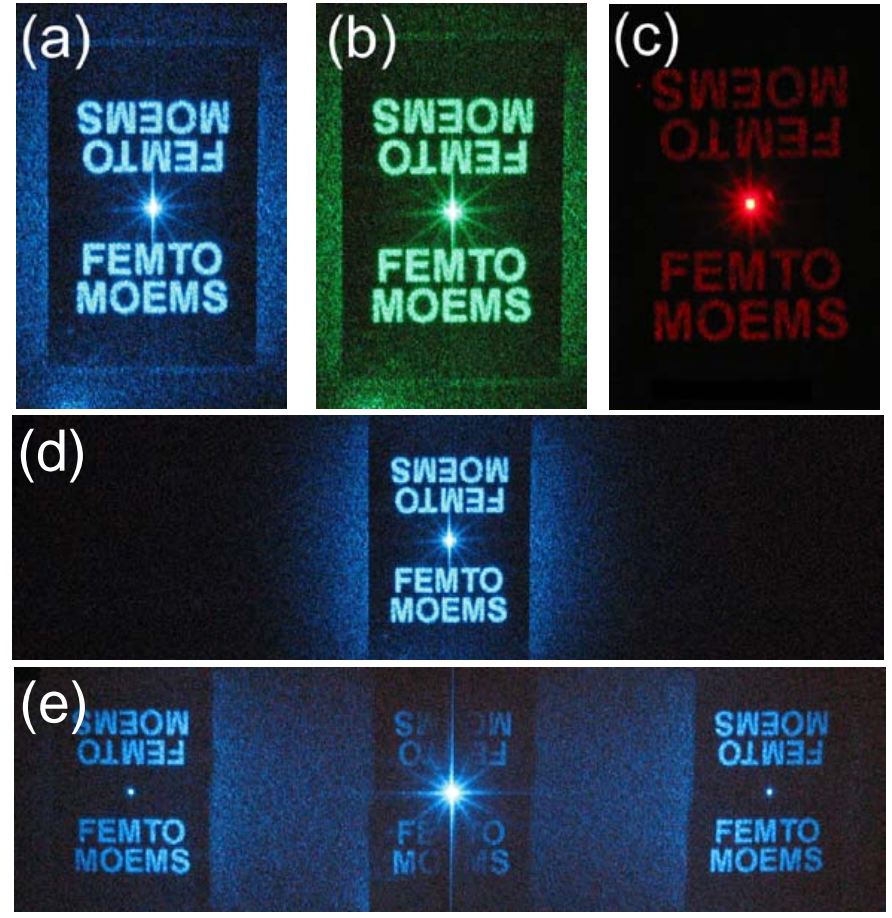

FIG. 10 Central area of the reconstruction of the kinoform CGH for (a) $488 \mathrm{~nm}$, (b) $543 \mathrm{~nm}$, (c) $633 \mathrm{~nm}$. (d) Wide reconstruction area from the kinoform CGH for $488 \mathrm{~nm}$. (e) The same as (d) but with the detour phase CGH.

optimized design based on an iterative Fourier transform algorithm, 2) its encoding either as a direct phase CGH or as a detour phase $\mathrm{CGH}, 3$ ) the $\mathrm{CGH}$ fabrication as a $\mathrm{SiO}_{2} / \mathrm{Si}$ profile, 4) the application of the spectroscopic ellipsometry technique to accurately determine the thickness of the $\mathrm{SiO}_{2}$ layer, 5) the use of the ellipsometric data to calculate the complex reflectance of the two levels in the hologram, 6) the optical inspection of the relative phase shift versus wavelength by means of a interferometric microscope and finally, 7) calculation of the diffraction efficiency and experimental evaluation of the quality of the hologram reconstruction.

The combined application of all these very well established techniques represents a useful guide for the production and inspection of $\mathrm{CGH}$ at relatively low cost. The developed $\mathrm{SiO}_{2} / \mathrm{Si} \mathrm{CGH}$ shows good quality on the reconstructed pattern, although a low efficiency due to the losses on reflection. However, they could be a good candidate to be used as a master CGH to be replicated directly with polymer, or onto $\mathrm{Ni}$ moulds by electroplating as inserts for mass production replication tools, such as hot embossing or micro-injection moulding [44].

\section{ACKNOWLEDGEMENTS}

IM and AMG acknowledge financial support from the Spanish Ministerio de Educación y Ciencia (grants FIS2009-13955C02-02).

\section{References}

[1] H. Herzig, Micro Optics (Taylor at Francis, London, 1997).
[2] S. Sinzinger, and J. Jahns, Microoptics (Wiley-VHC, Weinheim, 1999).

[3] B. Kress, and P. Meyrueis, Digital Diffractive Optics (John Wiley At Sons, Chichester, 2000).

[4] H. Kim, K. Choi, and B. Lee, "Diffractive optic synthesis and analysis of light fields and recent applications" Jpn. J. Appl. Phys. 45, 6555-06575 (2006).

[5] U. D. Zeitner, M. Banasch, and E.-B. Kley, "The making of a computer-generated hologram" Photon. Spectra 42, 58-61 (2008).

[6] L. P. Lesem, P. M. Hirsch, and J. A. Jordan, "The kinoform: a new wavefront reconstruction device" IBM J. Res. Dev. 13, 1503-155 (1969).

[7] A. J. Caley, M. Braun, A. J. Waddle, and M. R. Taghizadeh, “Analysis of multimask fabrication errors for diffractive optical elements" Appl. 0pt. 46, 2180-2188 (2007).

[8] D. C. O'Shea, J. W. Beletic, and M. Poutus, "Binary-mask generation for diffractive optical elements using microcomputers" Appl. 0pt. 32, 2566-2572 (1993).

[9] K. S. Urquhart, R. Stein, and S. H. Lee, "Computer-generated holograms fabricated by direct write of positive electron-beam resist" Opt. Lett. 18, 308-310 (1993).

[10] M. J. Verheijen, “E-beam lithography for digital holograms” J. Mod. Optic. 40, 711-721 (1993).

[11] H. C. Bolstad, T. Yatagai, and M. Seki, "Optimization of phase-only computer-generated holograms using an ion-exchange process" Opt. Eng. 31, 1259-1263 (1992).

[12] M. Flury, A. Benatname, P. Gérad, P. C. Montgomery, J. Fontaine, T. Engel, J. P. Schunck, and E. Fogarassy, "Excimer laser ablation lithography applied to the fabrication of reflective diffractive optics" Appl. Surf. Sci. 208, 238-244 (2003).

[13] M. T. Flores-Arias, A. Castelo, C. Gómez-Reino, and G. F. de la Fuente, "Phase diffractive optical grating on glass substrates by laser ablation" Opt. Commun. 282, 1175-1178 (2009).

[14] I. Moreno, C. Gorecki, J. Campos, and M. J. Yzuel, “Comparison of computer generated holograms produced by laser printers and lithography. Application to pattern recognition" Opt. Eng. 34, 35203525 (1995).

[15] C. Iemmi, S. Ledesma, J. Campos, and M. Villareal, "Gray-level computer-generated hologram filters for multiple-object correlation" Appl. Opt. 39, 1233-1240 (2000).

[16] I. Moreno, C. Iemmi, A. Márquez, J. Campos, and M. J. Yzuel, "Modulation light efficiency of diffractive lenses displayed onto a restricted phase-mostly modulation display" Appl. 0pt. 43, 62786284 (2004).

[17] A. W. Lohmann, and D. P. Paris, "Binary Fraunhofer holograms, generated by computer" Appl. 0pt. 6, 1739-1748 (1967).

[18] Y.-C. Chang, P. Zhou, and J. H. Burge, "Analysis of phase sensitivity for binary computer-generated holograms" Appl. Opt. 45, 42234234 (2006).

[19] P. Zhou, and J. H. Burge, "Fabrication error analysis and experimental demonstration for computer generated holograms" Appl. Opt. 46, 657-663 (2007).

[20] A. Márquez, J. Campos, M. J. Yzuel, I. Pascual, A. Fimia, and A. Beléndez, "Production of computer-generated phase holograms using graphic devices: application to correlation filters" Opt. Eng. 39, 1612-1619 (2000).

[21] S. Sinzinger, and V. Arrizón, "High-efficient detour-phase holo- 
grams" Opt. Lett. 22, 928-930 (1997).

[22] C. Haupt, M. Pahlke, R. Krupka, and H. J. Tiziani, "Computergenerated microcooled reflection holograms in silicon for material processing with $\mathrm{CO}_{2}$ laser" Appl. 0pt. 36, 4411-4418 (1997).

[23] K. Hedsten, A. Magnusson, J. Melin, P. Enoksson, J. Bengtsson, F. Nikolajeff, D. Karlén, H. Rödjegård, and G. Andersson, "Replication of continuous-profiled micro-optical elements for silicon integration" Appl. Opt. 45, 83-89 (2006).

[24] H. Fujiwara, "Principles of spectroscopic ellipsometry" in Spectroscopic Ellipsometry: Principles and Applications, 81-140 (John Wiley at Sons, Chichester, 2003).

[25] A. Bosseboeuf, and S. Petitgrand, "Interference microscopy techniques for microsystem characterization" in Optical Inspection of Microsystems, W. Osten, ed., 217-244 (CRC Press, Boca Raton, 2007).

[26] M. Skeren, I. Richter, and P. Fiala, "Iterative Fourier transform algorithm: comparison of various approaches" J. Mod. Optic. 49, 1851-1870 (2002).

[27] J. S. Liu, A. J. Caley, and M. R. Taghizadeh, "Symmetrical iterative Fourier-transform algorithm using both amplitude and phase freedoms" Opt. Commun. 267, 347-355 (2006).

[28] F. Wyrowski, "Diffractive optical elements: iterative calculation of quantized, blazed phase structures" J. 0pt. Soc. Am. A 7, 961-969 (1990).

[29] A. W. Lohmann, and S. Sinzinger, "Graphics codes for computer holography" Appl. Opt. 34, 3172-3178 (1995).

[30] S. N. Toma, A. Alexandrescu, D. Muller, R. Muller, M. Kusko, N. Dumbravescu, V. Nascov, and D. Cojoc, "Binary phase reflective diffractive optical elements. Design and fabrication" (International Semiconductor Conference CAS 2004, Sinaia, Romania, IEEE CAS Proceedings, 2, 401-44, 4-6 October, 2004).

[31] J. Albero, L. Nieradko, C. Gorecki, H. Ottevaere, V. Comez, H. Thienpont, J. Pietarinen, B. Päivänranta, and N. Passilly, "Fabrication of spherical microlenses by a combination of isotropic wet etching of silicon and molding techniques" Opt. Express 17, 6283-6292 (2009).

[32] J. A. Woollam, C. Bungay, L. Yan, D. Thomson, and J. Hilfinker, "Application of spectroscopic ellipsometry to the characterization of thin films" Proc. SPIE 4932, 393-404 (2003).
[33] C. M. Herzinger, B. Johs, W. A. McGahan, J. A. Woollam, and W. Paulson, "Ellipsometric determination of optical constants for silicon and thermally grown silicon dioxide via a multi-sample, multi-wavelength, multi-angle investigation" J. Appl. Phys. 83, 3323-3336 (1998).

[34] C. Gorecki, M. Józwik, and L. Salbut, "Multifunctional interferometric platform for on-chip testing the micromechanical properties of MEMS/MOEMS" J. Microlith. Microfab. 4, 041402 (2005).

[35] C.-C. Wang, J.-Y. Lin, H.-J. Jian, and C.-H. Lee, "Transparent thinfilm characterization by using differential optical sectioning interference microscopy" Appl. 0pt. 46, 7460-7463 (2007).

[36] M. Schulz, A. Wiegmann, A. Márquez, and C. Elster, "Optical flatness metrology: 40 years of progress" Opt. Pura Apl. 41, 325-331 (2008).

[37] J. N. Mait, A. Scherer, 0. Dial, D. W. Prather, and X. Gao, "Diffractive lens with features less than $60 \mathrm{~nm}$ " 0pt. Lett. 25, 381-383 (2000).

[38] J. Vukusic, J. Bengtsson, M. Chisoni, A. Larsson, C.-F. Carlström, and G. Landgren, "Fabrication and characterization of diffractive optical elements in InP for monolithic integration with surfaceemitting components" Appl. Opt. 39, 398-401 (2000).

[39] S. Tamulevicius, A. Goubiene, G. Janusas, A. Palevivius, V. Ostasevivius, and M. Andrulevivius, "Optical characterization of diffractive optical elements replicated in polymers" J. Microlith. Microfab. 5, 013004 (2006).

[40] A. Martínez, M. M. Sánchez-López, and I. Moreno, "Phasor analysis of binary amplitude gratings with different fill factor" Eur. J. Phys. 28, 805-816 (2007).

[41] J. W. Goodman, Introduction to Fourier Optics (2nd Edition, McGraw-Hill, New York, 1996).

[42] I. Moreno, J. Campos, C. Gorecki, and M. J. Yzuel, "Effects of amplitude and phase mismatching errors in the generation of a kinoform for pattern recognition" Jpn. J. Appl. Phys. 34, 6423-6432 (1995).

[43] J. E. Ford, F. Xu, and Y. Fainman, "Wavelength-selective planar holograms" Opt. Lett. 21, 80-82 (1996).

[44] J. Pietarinen, S. Siitonen, N. Tossavainen, J. Laukkanen, and M. Kuittinen, "Fabrication of Ni-shims using UV-moulding as an intermediate step" Microelectron. Eng. 83, 492-498 (2006). 